\title{
Author Correction: Bacterial variability in the mammalian gut captured by a single-cell synthetic oscillator
}

David T. Riglar (1), David L. Richmond (1), Laurent Potvin-Trottier (1), Andrew A. Verdegaal, Alexander D. Naydich, Somenath Bakshi (D), Emanuele Leoncini (D), Lorena G. Lyon, Johan Paulsson (1) \& Pamela A. Silver (D)

Correction to: Nature Communications https://doi.org/10.1038/s41467-019-12638-z, published online 11 October 2019.

In the original version of the Supplementary Information provided with this Article, Supplementary Table 1 incorrectly provided the details for strain LPT239 as "E. coli MC4100 + pLPT234 + pLPT145" and for strain LPT320 as “E. coli MC4100 + pLPT234 + pLPT41". The details for strain LPT239 should be "E. coli MC4100 + pLPT234 + pLPT41" and for strain LPT320 should be "E. coli MC4100 + pLPT234 + pLPT145". This has been corrected in the HMTL version of the article and the correct table appended below:

Original

Supplementary Table 1: Strains used in this study.

\begin{tabular}{|c|c|c|}
\hline Strain & Source & Details \\
\hline LPT239 & This study & E. coli MC4100 + pLPT234 + pLPT145 \\
\hline LPT320 & This study & E. coli $\mathrm{MC} 4100+$ pLPT234 + pLPT41 \\
\hline LPT322 & This study & E. coli MC4100 + pLPT234 + pLPT149 \\
\hline PAS715 & This study & E. coli MG1655 DE(lacI) DE(motA) rpsL K42R + pLPT234 + pLPT145 \\
\hline PAS716 & This study & S. Typhimurium LT2 + pLPT234 + pLPT145 (*streptomycin resistant through uncharacterized mutational selection). \\
\hline PAS717 & This study & E. coli Nissle $1917 \mathrm{DE}($ lacI $) \mathrm{DE}($ motA) rpsL K42R + pLPT234 + pLPT145 \\
\hline PAS718 & This study & E. coli MG1655 DE(lacI) DE(motA) rpsL K42R attTn7::pRNA1-mKate2 + pLPT234 + pLPT145 \\
\hline
\end{tabular}

Corrected:

Supplementary Table 1: Strains used in this study.

\begin{tabular}{|c|c|c|}
\hline Strain & Source & Details \\
\hline LPT239 & This study & E. coli $\mathrm{MC} 4100+\mathrm{pLPT} 234+\mathrm{pLPT} 41$ \\
\hline LPT320 & This study & E. coli MC4100+ pLPT $234+$ pLPT 145 \\
\hline LPT322 & This study & E. coli MC4100 + pLPT234 + pLPT149 \\
\hline PAS715 & This study & E. coli MG1655 DE(lacI) DE(motA) rpsL K42R + pLPT234 + pLPT145 \\
\hline PAS716 & This study & S. Typhimurium LT2 + pLPT234 + pLPT145 (*streptomycin resistant through uncharacterized mutational selection). \\
\hline PAS717 & This study & E. coli Nissle $1917 \mathrm{DE}($ lacI $) \mathrm{DE}($ motA $)$ rpsL K42R + pLPT234 + pLPT145 \\
\hline PAS718 & This study & E. coli MG1655 DE(lacI) DE (motA) rpsL K42R attTn7::pRNA1-mKate2 + pLPT234 + pLPT145 \\
\hline
\end{tabular}




\section{Additional information}

Supplementary information The online version contains supplementary material available at https://doi.org/10.1038/s41467-021-22149-5.

(c) Open Access This article is licensed under a Creative Commons Attribution 4.0 International License, which permits use, sharing, adaptation, distribution and reproduction in any medium or format, as long as you give appropriate credit to the original author(s) and the source, provide a link to the Creative Commons license, and indicate if changes were made. The images or other third party material in this article are included in the article's Creative Commons license, unless indicated otherwise in a credit line to the material. If material is not included in the article's Creative Commons license and your intended use is not permitted by statutory regulation or exceeds the permitted use, you will need to obtain permission directly from the copyright holder. To view a copy of this license, visit http://creativecommons.org/licenses/by/4.0/.

(C) The Author(s) 2021 